\title{
G036
}

\section{Application of Diffracted Wave Analysis to Time- lapse Seismic Data for CO2 Leakage Detection}

\author{
F. Alonaizi* (CO2CRC, Curtin University), R. Pevzner (CO2CRC, Curtin \\ University), A. Bona (CO2CRC, Curtin University), E. Caspari (CO2CRC, \\ Curtin University) \& B. Gurevich (CO2CRC, Curtin University / CSIRO)
}

\section{SUMMARY}

Time-lapse (TL) seismic technology has been utilized in $\mathrm{CO} 2$ geo-sequestration to verify the CO2 containment within the reservoir. A major risk associated with geo-sequestration is possible leakage of $\mathrm{CO} 2$ from the storage formation into upper layers. Therefore, the deployment of carbon capture and storage (CCS) projects requires reliable and fast detection of relatively small volumes of CO2 outside the storage formation. To do this, it is necessary to predict typical leakage scenarios and improve the subsurface leak-detection methods. The upward migrated $\mathrm{CO} 2$ will form small secondary accumulations with limited lateral extend. In contrast to petroleum exploration methods, which mostly utilise reflected waves, this spatially small $\mathrm{CO} 2$ amounts will produce seismic signals corresponding to diffracted waves. In this work, we present a technique that can enhance $\mathrm{CO} 2$ leakage detection based on diffracted wave analysis in time-lapses seismic data. Focusing on diffraction energy, we aim to image small objects with limited lateral extend, such as a plume containing with less than 10,000 tonnes of supercritical CO2. Using seismic forward modelling, we examine the sensitivity of the method to the amount of $\mathrm{CO} 2$ leaked into a formation. 


\section{Vienna ''11}

\section{Introduction}

Time-lapse (TL) seismic technology has been utilized in $\mathrm{CO}_{2}$ geo-sequestration to verify the $\mathrm{CO}_{2}$ containment within the reservoir. A major risk associated with geo-sequestration is possible leakage of $\mathrm{CO}_{2}$ from the storage formation into upper layers. Therefore, the deployment of carbon capture and storage (CCS) projects requires reliable and fast detection of relatively small volumes of $\mathrm{CO}_{2}$ outside the storage formation. To do this, it is necessary to predict typical leakage scenarios and improve the subsurface leak-detection methods.

The upward migrated $\mathrm{CO}_{2}$ will form small secondary accumulations with limited lateral extent. In contrast to petroleum exploration methods, which mostly utilise reflected waves, this spatially small $\mathrm{CO}_{2}$ amount will produce seismic signals corresponding to diffracted waves.

In this work, we present a technique that can enhance $\mathrm{CO}_{2}$ leakage detection based on diffracted wave analysis in time-lapses seismic data. Focusing on diffraction energy, we aim to image small objects with limited lateral extent, such as a plume containing with less than 10,000 tonnes of supercritical $\mathrm{CO}_{2}$. Using seismic forward modelling, we examine the sensitivity of the method to the amount of $\mathrm{CO}_{2}$ leaked into a formation.

\section{The method}

Landa and Keydar (1998) proposed a method designed to detect small objects using diffracted waves. By analysing the diffracted wave field, this method produces a so-called D-section (diffraction section) that contains only diffracted energy. In the context of $\mathrm{CO}_{2}$ leakage, we are interested in imaging the diffracted waves that are induced by the presence of a small object, i.e. the $\mathrm{CO}_{2}$ plume. Using TL approach, we can acquire seismic data before and after $\mathrm{CO}_{2}$ injection, cross-equalise these two data sets, and subtract them from one another, thus removing the seismic response of any constant geological structure. This process should illuminate the diffracted waves produced by the $\mathrm{CO}_{2}$ plume and separate them from the reflections which are dominant in seismic data. The algorithm used to detect the diffractors is based on measuring the coherency of the data by using the semblance function $S$ with normalized input to output energy ratio (Taner and Koehler 1969),

$$
S=\frac{1}{N} \frac{\sum_{t=-m}^{+m}\left(\sum_{i} f_{i t(i)}\right)^{2}}{\sum_{t=-m}^{+m}\left(\sum_{i} f_{i t(i)}{ }^{2}\right)},
$$

where the inner summation of the sample $f_{i t i}$ is performed for all the traces (index $i$ ) at a given time (index $t$ ), and the outer summation is performed over a given time window of width $2 m(N$ is the number of channels). Semblance $S$ is computed along the traveltime curve of the diffracted wave. This will focus the energy back to the apex of the diffraction hyperbola at the location of the plume using the following equation:

$$
t_{x}=\sqrt{t_{\circ}^{2}+\frac{4 x^{2}}{v^{2}}}
$$

where $x$ is the lateral distance from the diffractor, $t_{0}$ is the two-way traveltime at the apex, and $v$ is the NMO velocity obtained from the velocity analysis. The input data to compute the D-section is the difference stack section that is expected to contain only the diffracted wave energy produced from the $\mathrm{CO}_{2}$ plume. 


\section{Vienna '11}

\section{Seismic forward modelling}

We created two different 2-D depth models simulating the stage 2 of the CO2CRC Otway Project. This stage includes $\mathrm{CO}_{2}$ injections into saline aquifer, the Paaratte formation, at around $1500 \mathrm{~m}$ depth and aims to understand the $\mathrm{CO}_{2}$ behaviour in saline formations. The models consist of thirteen layers with a small plume added into saline aquifer at depth $\sim 1.5 \mathrm{~km}$ as shown in Figure 1(a). The synthetic datasets were generated using $50-\mathrm{Hz}$ Ricker wavelet. The velocities are based on the interval velocities calculated from zero-offset VSP data acquired at Naylor-1 monitor well (Pevzner et al. 2009). The fluid substitution modeling was performed to calculate the physical properties of the host formation in order to predict the change of the acoustic/elastic properties of the rock after injection, i.e. $V_{P}, V_{S}$, and density, resulting in the following values:

- Pre-injection: $100 \%$ brine, $V_{P}=3375 \mathrm{~m} / \mathrm{s}, V_{S}=1801 \mathrm{~m} / \mathrm{s}, \rho=2322 \mathrm{~kg} / \mathrm{m} 3$.

- Post-injection: $70 \%$ brine and $30 \%$ gas, $V_{P}=3164 \mathrm{~m} / \mathrm{s}, V_{S}=1821 \mathrm{~m} / \mathrm{s}, \rho=2172 \mathrm{~kg} / \mathrm{m} 3$.

From the flow modelling and assuming uniform saturation, we conclude that the plume size corresponding to $10,000 \mathrm{t}$ of $\mathrm{CO}_{2}$ is $170 \mathrm{~m}$ wide with the thickness of about $13 \mathrm{~m}$. Our rock-physics modelling shows that $\mathrm{CO}_{2}$ has an effect on seismic velocity. However, poor data condition can significantly reduce the TL signal. Therefore, to simulate real data conditions, we performed the following steps:

- Contaminate the data with random noise with the same amplitude spectra as for the seismic wavelet. The amount of noise added is sufficient to provide the same level of repeatability (NRMS 30\%) as observed on the real data (Pevzner et al., 2011). Here we assume that presence of this noise is the only factor controlling the repeatability.

- Correct the plume amplitude response obtained from the 2D finite deference modelling to 3D situation.

- Downscale the amplitude of the diffracted waves in proportion to the $\mathrm{CO}_{2}$ volume.

\section{Results and discussion}

We produced two stack sections, the baseline (without plume) and monitor section (with a $\mathrm{CO}_{2}$ plume). The same amounts of noise were added to both sections. The noise has a significant effect on the diffracted waves, as shown in Figure 2 (b); this indicates that the diffraction energy is smaller and more sensitive to noise than the reflection energy. Therefore, dip moveout correction (DMO) has been applied to the data to enhance the diffracted wave energy, and thus to improve the detection.

In the noise free section, Figure 2 (a), we can see two diffraction events close to each other caused by the edges of the plume. We can observe a phase reversal between the two sides of the diffraction traveltime curve. This result is consistent with the work of Trorey (1970) and has been observed on field data as well (Harlan et al. 1983). Thus, applying equation (1) to both sides of the diffraction curve reduces the coherency of the signals due to 180 degree phase change at the apex of the hyperbola. Therefore, we produced two D-sections computed along each side of the diffraction hyperbola and then added them together. This process enhances the energy on each side of the plume by avoiding amplitude cancelation during the correlation process. Figure 3 shows a comparison between a $D$-section computed along both sides of the diffraction hyperbola (a), and D-sections computed separately for each side of the hyperbola and then added together (b); the latter section has better result and contains less noise.

In order to predict the minimum amount of $\mathrm{CO}_{2}$ that is possible to detect based on our data conditions, we produced a number of stack sections corresponding to plumes containing different amounts of $\mathrm{CO}_{2}$. Figure 4 shows the $D$-section (b) computed from a stack section (a) with 2,000 tof $\mathrm{CO}_{2}$ plume. The $\mathrm{CO}_{2}$ plume is depicted on the D-section as an anomaly, and can be easily identified. The minimum amount of $\mathrm{CO}_{2}$ detectable from the data is about 2,000-3,000 tons. For less than 2,000 t of 


\section{D'vienna |'11}

$\mathrm{CO}_{2}$, the diffraction amplitude becomes too weak and cannot be reliably detected with the presented method.

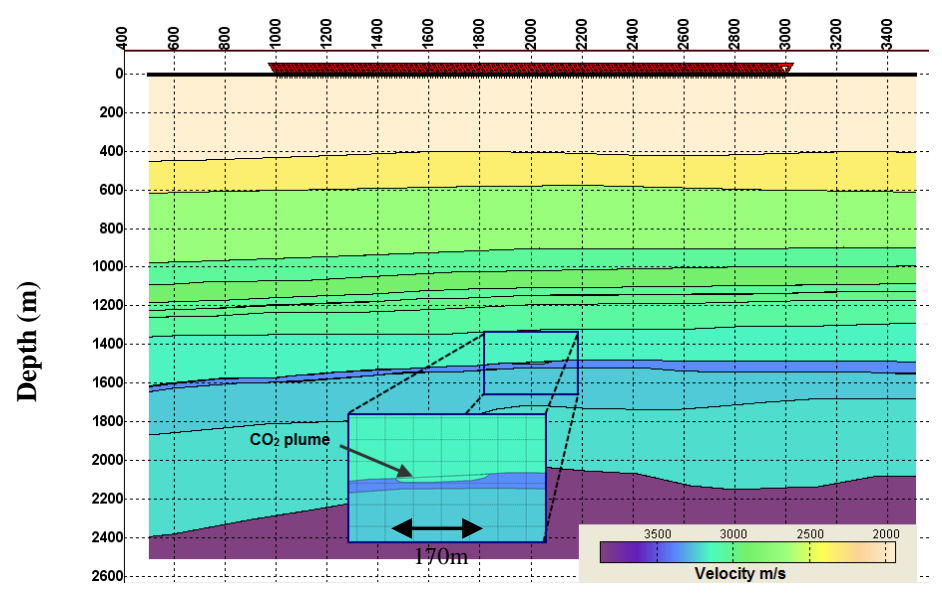

Figure 1 (a) A velocity depth model created for the experiment, the plume with $170 \mathrm{~m}$ length and $13 \mathrm{~m}$ thick added in the monitor section.
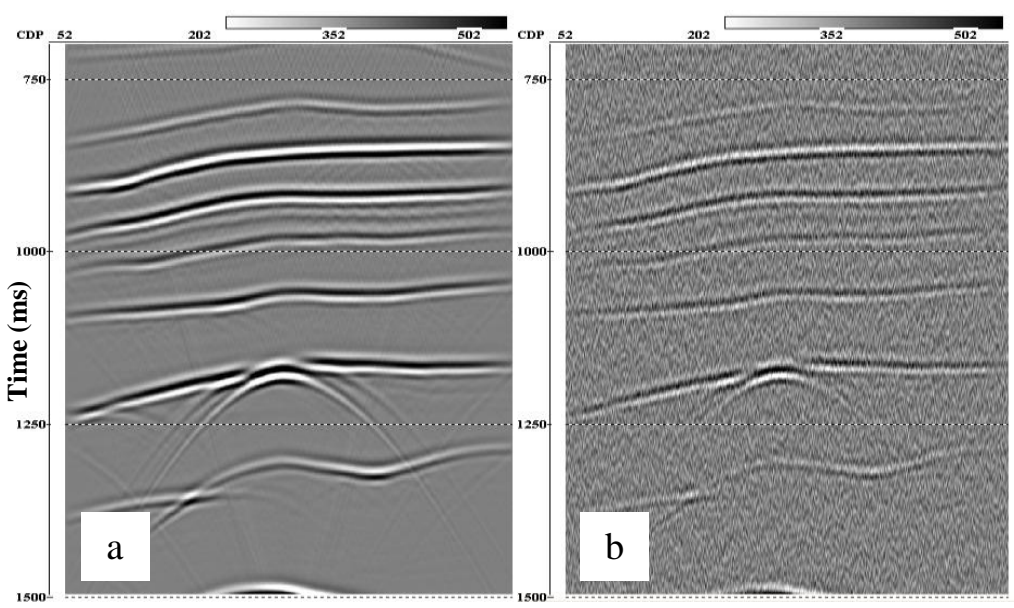

Figure 2 (a) A part of the stack sections of the geological model, (b) the same section after adding noise. Note how the noise is affecting the diffracted energy.
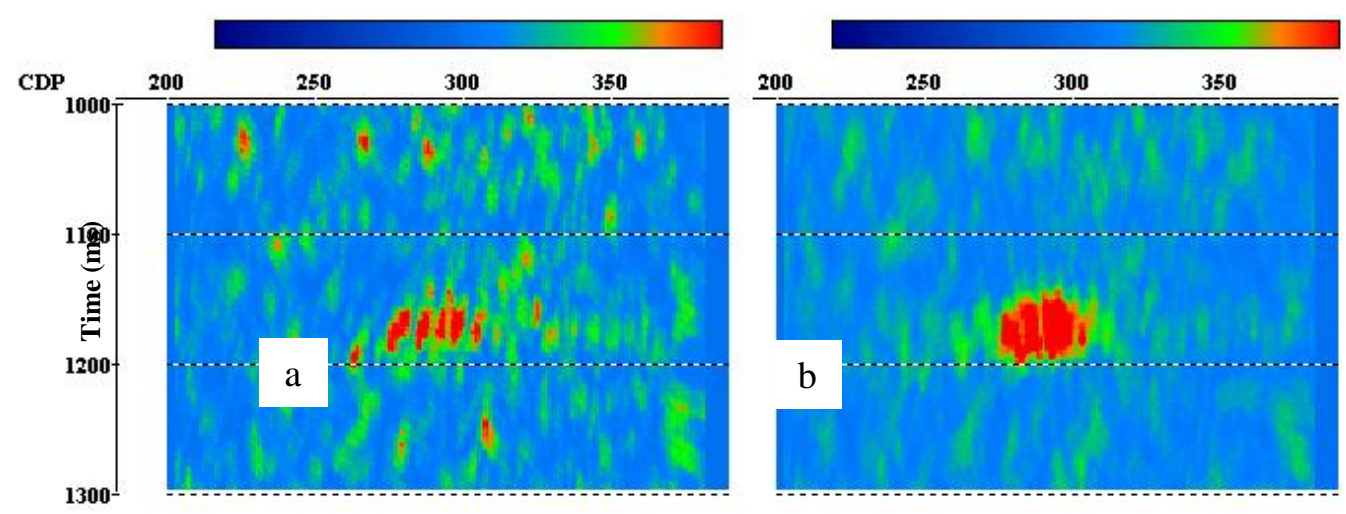

Figure 3 A comparisons between two D-sections (a) computed over the full diffraction traveltime curve, and (b) a combined two D-sections computed over each side separately. Note on (b) the plume shows stronger anomaly and the noise is significantly suppressed. 


\section{Vienna |'11}

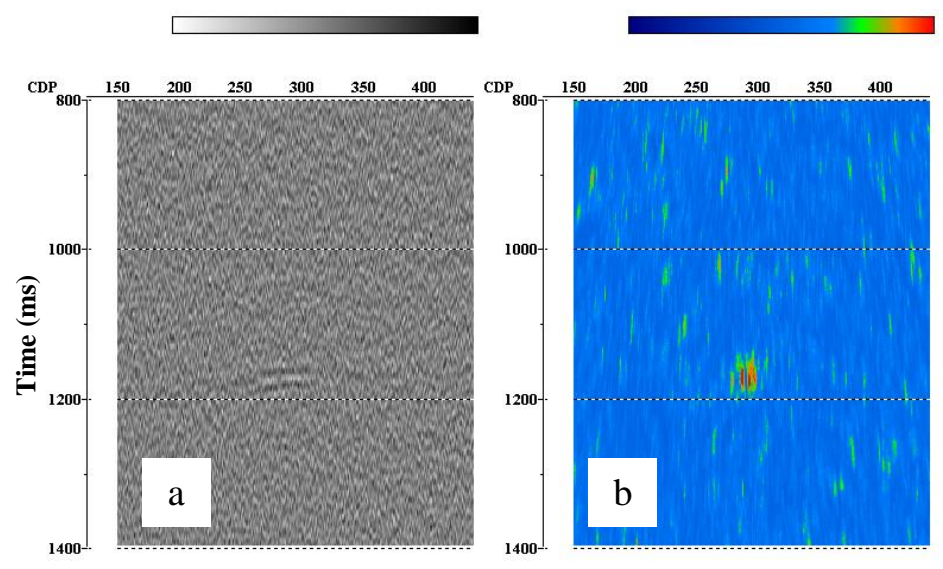

Figure 4 A D-section (b) computed from TL stack section (a) of 2,000 tones of CO2 plume.

\section{Conclusions}

We presented a technique that has the ability to image small $\mathrm{CO}_{2}$ volumes from seismic data with low $\mathrm{S} / \mathrm{N}$ ratio by measuring the coherency (semblance) along the diffracted-wave traveltime. The method has been applied to post-stack data, using the velocity field (the stacking velocity) which makes computing $D$-sections robust. Using TL data analysis, we are able to isolate the diffracted wave from other wavefield components. Applying the method along each half of the diffraction hyperbola and then combining them avoids amplitude cancelation. We found that the amplitude of the diffracted waves is much weaker than the amplitude of the reflected waves, which makes the diffracted events more sensitive to noise and difficult to detect on seismic data. Applying DMO to the data enhances the diffracted waves and reduces the noise on D-sections. From the synthetic data based on the geology of the CO2CRC Otway test site, the predicted minimum detectable amount of $\mathrm{CO}_{2}$ plume is about 2,000 to 3,000 tones. The use of diffractions rather than reflections for monitoring small amount of $\mathrm{CO}_{2}$ can enhance subsurface monitoring and detect relatively small amounts of $\mathrm{CO}_{2}$.

\section{Acknowledgements}

This work is funded by the Commonwealth of Australia through CO2CRC research activities. We thank Dr Evgeny Landa for the useful advice and Dr Yildiray Çinar for the flow simulation results.

\section{References}

Harlan, W. S., Claerbout, J. F. and Rocca, F. [1983] Extracting velocities from diffractions. SEG Technical Program Expanded Abstracts 2(1), 574-577.

Landa, E. and Keydar, S. [1998] Seismic monitoring of diffraction images for detection of local heterogeneities. Geophysics 63(3), 1093-1100.

Pevzner, R., Shulakova, V., Wisman, P. and Urosevic, M. [2009] Seismic Monitoring Feasibility Study of CO2 Injection into Saline Aquifer - A Case Study from Otway Basin, Australia. $71^{\text {st }}$ EAGE Comnference \& Exhibition, Extended abstract.

Pevzner, R., Shulakova, V., Kepic, A. \& Urosevic, M. [2011] Repeatability analysis of land timelapse seismic data: CO2CRC Otway pilot project case study. Geophysical Prospecting, 59, 6677.

Taner, M. T. and Koehler, F. [1969] Velocity spectra---digital computer derivation applications of velocity functions. Geophysics 34(6), 859-881.

Trorey, A. W. [1970] A simple theory for seismic diffractions. Geophysics 35(5), 762-784. 